\title{
Children first
}

\author{
Drugs administered to children with cancer were typically developed under the assumption that childhood cancers \\ are similar to their tissue-matched adult counterparts. Focusing on identifying and targeting alterations present \\ specifically in childhood tumors will accelerate the development of tailored therapies and improve the prognosis of \\ children with cancer.
}

S ince the passing of the Pediatric Research Equity Act in 2003, the development of a plan for treating children using drugs developed for adults has been mandated in the US, but, in fact, molecularly targeted cancer agents have been exempt from this requirement. Similarly, the European Medicines Agency (EMA) has also required that companies requesting approval for a new medicine provide the agency with a pediatric investigation plan (PIP). Companies can request a waiver, however, and have done so when they consider their 'adult' cancer drug not to be applicable to children. On 3 August in the US, the Research to Accelerate Cures and Equity (RACE) for Children Act was passed through the Senate. This act has now been signed by President Trump, and will mandate, without exemption, that companies test molecularly targeted cancer drugs in pediatric as well as adult patients if the target is considered to be relevant in that childhood cancer. This is a step in the right direction, but the development of these drugs could also be pushed harder if coordinated with a drive to further understand the mechanisms of childhood cancer.

Childhood cancer death rates have declined in the US by approximately 20\% between 1999 and 2014, and yet the negative side effects of currently available therapies for young patients with these malignancies raises the urgency for developing newer, targeted medicines for them. Radiation in pediatric patients with brain tumors is linked to neurocognitive decline and induces other tumors later in life, as highlighted in a recent study that was able to pinpoint the cancer-causing mutations that resulted in radiation-induced meningiomas (Nat. Commun. 8, 186, 2017). Chemotherapy can have equally detrimental late effects, including on the brain and hearing. Being able to combine chemotherapy with a targeted therapy may enable reductions in the dosage of chemotherapy needed for efficacious treatment.

But there is currently a very 'adult first' approach to developing new therapies for childhood cancer. Many of the drugs available for pediatric cancer trials are being repurposed, such as panobinostat for diffuse intrinsic pontine glioma (see go.nature.com/2wx18tc). This is because childhood cancers have typically been viewed as being similar to their adult-tissue counterparts. A more tissue-agnostic, target-centric approach to the development of new therapies would make the point that these therapies may be relevant to some pediatric as well as adult cancers. EMA regulators and companies are actively discussing the possibility of adopting this type of gene-based strategy for the PIP. Encouragingly, in May, the US Food and Drug Administration (FDA) approved pembrolizumab for use against both adult and pediatric solid cancers on the basis of specific molecular characteristics.

The flow of information from adult-drug development to pediatricdrug development as above must also be complemented by more pediat- ric-cancer-centric approaches. The development of effective new therapies for pediatric cancers also requires that scientists understand the unique causes and mechanisms involved in their genesis and spread. Recent genomic characterizations of pediatric cancers, however, have identified a paucity of such genetic alterations when compared to adult cancers. Fusion proteins have often been identified as singular drivers in pediatric cancers, and they seem to work by deregulating gene-regulatory complexes such as transcription factors. That tumors in children differ in some way to adult tumors should probably not come as a surprise: because the former occur so early in life, they are less likely to form as a result of environmental mutagens or accumulations of errors of replication.

In addition to the unique genetic mechanisms at work in childhood cancers, the cell of origin of pediatric cancers seems to provide important clues as to how they originate and develop. In the US, the two most common types of childhood cancer are leukemias and brain tumors. Thus, it seems that the specific cellular context in which the few mutations that are found in these cancers operate-notably, the stage of cell development-affect tumorigenesis (Cell 166, 1132-1146, 2016). One way to target these cancers might, therefore, be by specifically targeting the microenvironment that provides signals that regulate developmental processes, and hence, these cancers. Importantly, however, one must avoid targeting active processes that are fundamental to the development of healthy tissues that continue throughout childhood and puberty; for example, therapies targeting neurogenesis could potentially be more toxic than chemotherapy in the long term. That this will necessitate extensive follow up after childhood cancer drug trials to observe any long-term harmful consequences is also not an unimportant point. How best to combine such developmentally targeted therapies with the current standard of care could also be a challenge to figure out.

In the US, the Cancer Moonshoot pediatric working group has recently provided a framework for the characterization and development of model systems for childhood cancer, in particular, the development of cellular and animal models of the more recently identified gene fusions. Furthermore, in August, the NIH Common Fund announced that the Gabriella Miller Kids First Pediatric Research Program will work on making a new data resource available that will provide a centralized genetic database of pediatric cancers and structural birth defects. These are both steps in the right direction, and considering the rarity of many pediatric cancers, such consortia that collect longitudinal data of molecular features of tumors, as well as their clinical features, will be essential for furthering our understanding of childhood cancer. Making sure that childhood cancers, as with adult cancers, are fully characterized and receive the attention that they deserve will accelerate the development of much-needed targeted therapies. 